\title{
Identification Methods of Underwater Noise Sources Generated by Small Ships
}

\author{
I. GLOZA* \\ Polish Naval Academy, Śmidowicza 69, 81-919 Gdynia, Poland
}

\begin{abstract}
In this work we were interested in the use for the localization of the ship noise sources in the water environment. Particular interest was dedicated to identifying of narrow band contribution to the total spectra of the ship hydroacoustic noise. The analyses were performed in time and frequency domain. The diesel engine, the electric motor and the propeller have notably been studied and the different frequencies connected to these devices have been listed. Extensive measurements were made of the underwater radiated noise of $\mathrm{K} 1$ and K2, small vessels powered by an electric and diesel engines. The discrete Fourier transform and the short time Fourier transform and sound intensity method were used for a precise analysis. The coherence function was also performed to associate each component of underwater noise with the vibrating part of the engine, which generates it. The measurement results obtained here have been later analyzed and a criticism of each method has been done, focusing on its advantages in the field of the identification of the noise sources.
\end{abstract}

PACS: $43.30 . \mathrm{Nb}$

\section{Introduction}

Hydroacoustics is a branch of technical sciences which incorporates many disciplines, such as physics (acoustics), signal analysis, electronics, sensors etc. These branches are widely used in planning and realization of not only numerous military systems, but also non-military applications. Weapons like sea mines and torpedoes use different sensors to detect, classify and localize targets. To deal with this threat, ship underwater signatures are key elements of naval platform designs and operations.

Radiated noise from ships can be an important contributor to the Baltic Sea ambient noise, so near ports ship noise is a major part of shallow water noise environment. Ship noise control and reduction is a significant factor in the performance of underwater acoustic systems and in the habitability of the vessels for the crew and sometimes passengers.

Ship noise spectra are usually classified in two types: broadband noise having a continuous spectrum of cavitation and tonal noise which is full of discrete noise frequency or line components related to machinery, propellers, generators, gears, etc.

One of the principal objectives of this paper is to provide engineering procedures for estimating ship machinery sources and propeller noise. Underwater sources in the absence of cavitation are above all dipoles and are dominated by vibration. Flow-induced sound from underwater sources differs from the features of aerodynamically radiated sound, this is because of the low mach

\footnotetext{
* e-mail: i.gloza@amw.gdynia.pl
}

numbers of single-phase hydrodynamic flows, which make these canonical sources very unproductive. Cavitation and bubbly flows are among the most efficient of hydrodynamic sources because these are monopoles and have matching parts in subsonic combustion flow aeroacoustics. Propulsion propellers, that represent major sources of ship noise and similar to helicopters with rotating blades operating in nonuniform flow fields, are the subject of continuing noise (continuous spectrum).

After WW II Urick [1] studied the ship underwater noise in the US Navy, after that Ross [2] measured noise of some big commercial vessels and described trends in ship sizes and powering. Also in Europe (France, Germany, Norway, Sweden, Finland and Poland) many researchers investigated ship noise and vibration. For example, a modern acoustic test range is located at Heggernes, near Bergen, Norway, which is used for measuring the underwater noise from all types of NATO warships and also some important civilian vessels [3]. In the 1980s new theoretical and practical researches on ship noise prediction was undertaken by Polish scientific workers from Naval Academy of Gdynia.

\section{Methods of measurements}

The measurements were carried out in the Measurement and Control Acoustic Ranges (MACARs), which is located in the southern part of the Baltic Sea. The MACARs are approximately $20 \mathrm{~km}$ long by $8 \mathrm{~km}$ wide and the depth of the water over the area varies between 5 to $20 \mathrm{~m}$. This range contains a precise noise measurements system consisting of bottom-mounted hydrophones. Use of such system eliminated much of the low-frequency wave-induced noise. These detectors are 
Brüel \& Kjær and RESON production. The hydrophones were calibrated to frequencies as low as $5 \mathrm{~Hz}$. The signal-to-noise ratio for the spectrum data was at least $20 \mathrm{~dB}$. The best location of the acoustic range is that where the ambient noise is the smallest and the depth of sea is enough high that the bottom is reflectionless one. Therefore the hydrophone array was a bottom-mounted setup.

The runs were recorded by means of the multichannel digital recorder (PULSE). The narrow-band power spectra in $0.5 \mathrm{~Hz}$ bands were achieved from $8 \mathrm{~s}$ samples. Sometimes the bandwidth of spectra was $0.125 \mathrm{~Hz}$, which was obtained by uniform sampling of an $8 \mathrm{~s}$ noise recorded at $8192 \mathrm{~Hz}$.

At the same time, on-board measurements of the vibration of the diesel and electric engines were carried out. Because of this small depth not only pressure, but also sound intensity are often measured. This area is relatively sheltered and free from sea and air interference. The best location of the measurement range is that where the ambient noise is the smallest and the depth of the sea is enough high that bottom is not including reflections [4].

During the ship measurements the conditions were good, the wave height was less than $0.5 \mathrm{~m}$ and wind speeds less than $5 \mathrm{~m} / \mathrm{s}$, therefore the ambient noise level was low. The sound speed profiles at the time of the experiment were typical of the early autumn or the late summer: an even, gradually decreasing gradient without mixed layers.

The signals used in this experiment quickly change with time, the discrete Fourier transform (DFT) is not suitable for a precise analysis. It gives no information on the spectrum evolution in time. However a second type of the Fourier transform can be used in this case: the short time Fourier transform (STFT). To calculate the spectrum of signal, the STFT uses a finite time window which scans the whole signal. This window makes a hypothesis of signal stationary possible. The main advantage of this method is that it gives the temporal evolution of the signal's spectrum. However, STFT disadvantage is that the frequency resolution is linked to time resolution. It is not possible to have high resolution in time and frequency at the same time. A tool which makes the calculation of a signal's spectrum in a frequency bandwidth with a specified resolution possible is the chirp- $z$ transforms (CZT). This process makes a zoom on the spectrum.

The sound intensity probe is designed to capture the sound pressure together with the unit direction of flow as a vector quantity. This is achieved by setting up more than one hydrophone in a probe to measure the sound energy flow. The main advantages of vector sound-intensity measurements are: first - determining which parts of the complex source radiated most noise and second finding the directions of sound sources. Therefore noise sources localization onboard is of the highest importance for underwater acoustic. In that way sound intensity probes are used to give the direction and the magnitude of sound sources $[5,6]$.

\section{Results of research}

We have used, as mentioned above, these different methods for the identification of the noise sources onboard two small vessels. For simplicity the ships are called $\mathrm{K} 1$ and $\mathrm{K} 2$. It must be said that $\mathrm{K} 1$ and $\mathrm{K} 2$ are the ships of the same project. Ships K1 and K2 have both electric motors (linked with propeller's shaft) and diesel motors, whose task is generating electricity for electric motors (they are not linked with propeller's shaft). Their acoustic field measurements are made during numerous sea trails at several speeds, from 1.7 to $9.8 \mathrm{kn}$, and for two power configurations, electric and diesel.

The simplest method of all these described in this part is measuring the sound pressure level (SPL). On the other hand, it gives some useful pieces of information. It is useful to compare the noise from different vessels and also the ship noise with the level of the ambient noise because these are directly linked with the sound pressure levels.

During ship runs its propeller blade passes through the region of low pressure. That generated sound in form of the pulsation of cavity can be approximated by a monopole source. This process takes place in the vicinity of the free-pressure surface so the nearly ideal reflections of the sound waves occur as the second source. In result of these processes the radiation pattern of the propeller noise has a dipole character with a dipole directivity pattern.

Contrary to the pressure level, the sound intensity level points out precisely the different sources of noise. Figure 1 displays the sound intensity level for $\mathrm{K} 1$ at the speed of $6 \mathrm{kn}$.

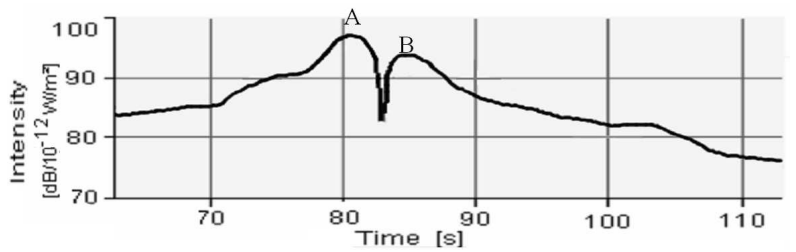

Fig. 1. Sound intensity level for $\mathrm{K} 1 \mathrm{with}$ diesel at $6 \mathrm{kn}$.

In the curve it can be noticed that the ship is above the probes at $82 \mathrm{~s}$. But the most interesting information is two local maxima at $80 \mathrm{~s}$ and $85 \mathrm{~s}$. They correspond respectively to the diesel noise (A) and to the propeller noise (B). It is important to say that for this speed the main source is the diesel engines noise which is higher than the electric motor and the propeller noises. On the other hand, when the ship is running above the critical speed the noise generated by the propeller is higher than the noise created by the machinery $(\mathrm{B}>\mathrm{A})$. This is due to the strong cavitation of the propeller.

Most of the information about the ship noise and its vibration is of course located between 0 and $200 \mathrm{~Hz}$, so the bandwidth used for the analysis was reduced to $200 \mathrm{~Hz}$. 
Thanks to this reduction many details are visible on the spectrum.

Below there are shown the spectra of the K1 running at $6 \mathrm{kn}$, in green, and $7.8 \mathrm{kn}$, in red, where the black and green circled peaks on the red curve respectively correspond to the fundamental and its second harmonic. To improve the clarity of this figure the pressure level of the red one was shifted up at $10 \mathrm{~dB}$ which explains the irregular values.

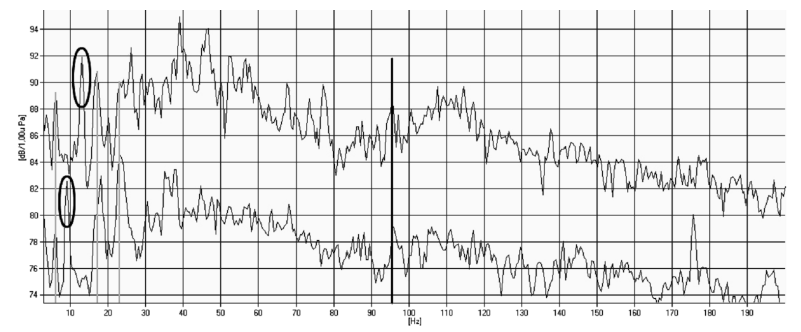

Fig. 2. Spectra of $\mathrm{K} 1$ running at $6 \mathrm{kn}$ (lower) and $7.8 \mathrm{kn}$ (upper).

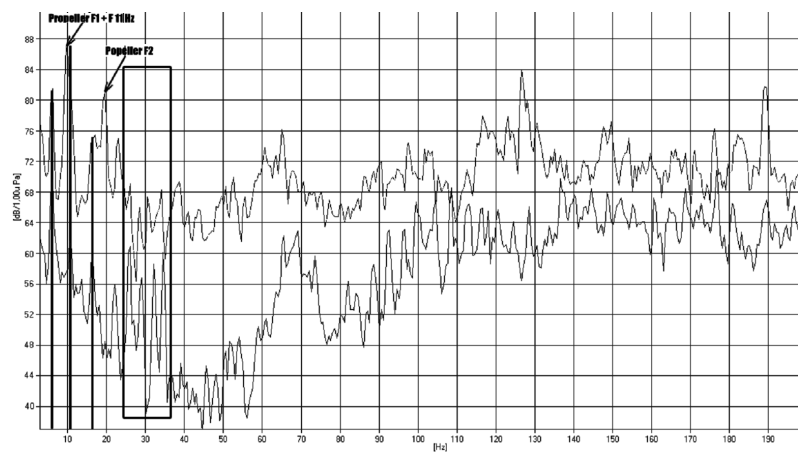

Fig. 3. Spectra of K2 running at $1.8 \mathrm{kn}$ (lower) and $6 \mathrm{kn}$ (upper).

It is necessary to distinguish the stable frequencies from the mobile vessels. Contrary to the shaft and the propeller which have varied rotational speeds, some gears run at a constant speed. The mere fact of knowing the rotational speed almost makes the immediate specifying of the source possible. In Fig. 2, four main frequencies can be highlighted: $6.5,17,23$ and $95 \mathrm{~Hz}$. The $0-30 \mathrm{~Hz}$ bandwidth and the remaining frequency, marked in black ellipse, is movable. It can be easily asserted that the latter one is due to the rotation of the propeller.

Now let us see the results of K2 sounds analysis (Fig. 3). The propeller frequency is the dominant one of the spectrum. This is observable when $\mathrm{K} 2$ runs at $6 \mathrm{kn}$; the fundamental reaches a level of $88 \mathrm{~dB}$ with the contribution of only one peak at $11 \mathrm{~Hz}$. The $6.5 \mathrm{~Hz}$ frequency also appears on every spectrum and its amplitude is strongly connected with the speed of the vessel. When running at $1.8 \mathrm{kn}$, the level of this peak is $75 \mathrm{~dB}$ where as it is $95 \mathrm{~dB}$ for a speed of $9 \mathrm{kn}$. This last statement allows to stress that the 6.5 frequency is due to the ship presence and not the environment. Finally, the $17 \mathrm{~Hz}$ and the $23 \mathrm{~Hz}$ frequencies are present too, but the latter seems less dominant and stable than for K1. It is interesting to notice that four grouped peaks $(26,28.5,32$ and $34 \mathrm{~Hz}$ ) occupy only the $25-35 \mathrm{~Hz}$ narrow bandwidth.

These are very stable in frequency when the speed increases. It is hard to say whether there are fundamentals among them but it is sure that their amplitudes are affected by harmonics, as the contribution of the $17 \mathrm{~Hz}$ second harmonic on the $34 \mathrm{~Hz}$ peak, shows.

Above the $50 \mathrm{~Hz}$ frequency, some peaks appear. They correspond to the harmonics from 0 to $40 \mathrm{~Hz}$ bandwidth. For example, the vertical black line at $95 \mathrm{~Hz}$, in Fig. 2, marks the fourth harmonic of $23 \mathrm{~Hz}$ frequency for $\mathrm{K} 1$.

To compare with the electric configuration, the diesel engine is definitely the noisiest. It only takes one look at the spectra between the two different workings to become aware of the diesel indiscretion. Moreover their shapes are very different as the following Fig. 4 shows.

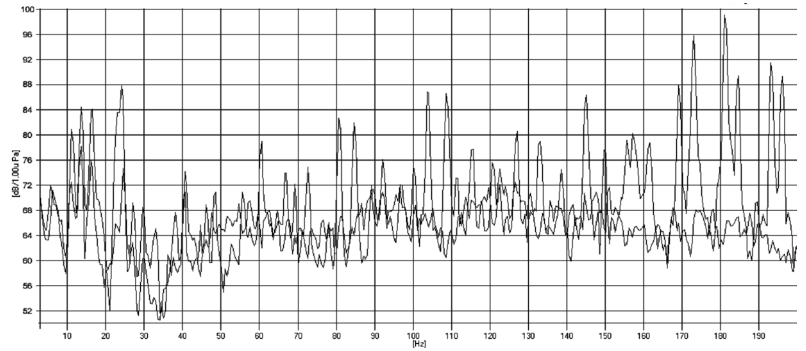

Fig. 4. Spectra of two working engines (diesel and electric) for $\mathrm{K} 2$ running at $7.1 \mathrm{kn}$.

It is not possible to be mistaken between diesel and electric power configurations. In addition, listening to the audio recordings shows that a high-pitched sound is present for the electric motor contrary to the diesel one where the thumping noise from the engine is dominant. The most striking is the number of peaks and their amplitudes. The peaks exceed the average level by $20 \mathrm{~dB}$. Of course the discrete frequencies of the propeller are still present but are not dominant anymore. The following figure displays the structure of the spectrum of K1 at $6 \mathrm{kn}$ in diesel configuration. The diesel engines are used for electric supply with a speed between 1400 and $1500 \mathrm{rpm}$. Therefore the structure of their spectrum is always the same.

From Fig. 5, the frequency of the diesel firing rate (explosions) can be extracted thanks to the study of the peak amplitudes. At the $143 \mathrm{~Hz}$ frequency the contribution of the fundamental of the explosions can be observed. Without this contribution, the peak should reach the value of $84 \mathrm{~dB}$ - where the third black slanting line crosses the green peak - and yet it exceeds it by $3 \mathrm{~dB}$. As the diesel engines turn at $1440 \mathrm{rpm}$, it results in a frequency of the explosions of $144 \mathrm{~Hz}$ in agreement with the observation. In some measurements weak tones at the intermediate half-firing rate frequencies are also identified. 


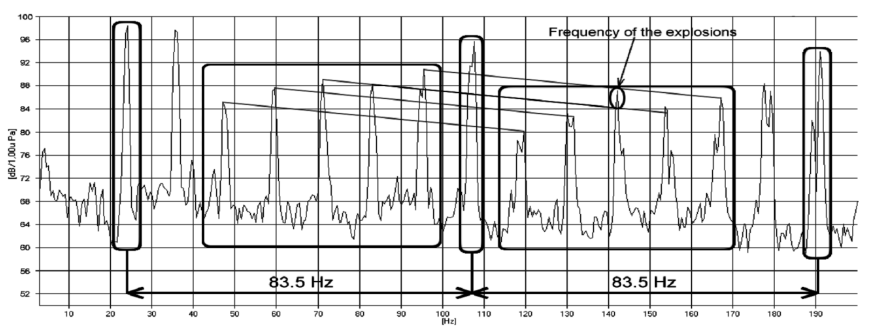

Fig. 5. Spectrum between 0 and $200 \mathrm{~Hz}$ of $\mathrm{K} 1$ running at $6 \mathrm{kn}$.

Three main peaks - marks by arrows - stand out from the ordinary ones as far as amplitude is concerned. At $23.9,107.4$ and $190.9 \mathrm{~Hz}$ there are the first, the second and the third peak, whose levels are in order of the highest ones for the example of K1 running at $6 \mathrm{kn}$. In addition, $83.5 \mathrm{~Hz}$ separates them. At last, this frequency spacing seems to depend on the speed because from measurements we know that it is of $70.5 \mathrm{~Hz}$ for K1 running at $5 \mathrm{kn}$. There is an increase of $13 \mathrm{~Hz}$ for a $1 \mathrm{kn}$ speed which corresponds to an increase of $20 \mathrm{rpm}$. The black slanting lines which link two peaks together have the same incline which means that there is the same linear decline between those pairs of peak. Consequently those linked peaks surely are harmonics. Nevertheless, their sources are not as easy to define as the one of the propeller.

If the study of this spectrum is pursued, many sources get showed as the following Fig. 6 illustrates. Each letter contained in the black boxes corresponds to a source. Thus, in this case, there are 11 different sources in $0-150 \mathrm{~Hz}$ bandwidth. The corresponding levels are put in brackets on the right of the source. For each peak, the level and the frequency are written at the bottom of the figure. You will remark that the level of each peak is the sum of the different contributions of each box.

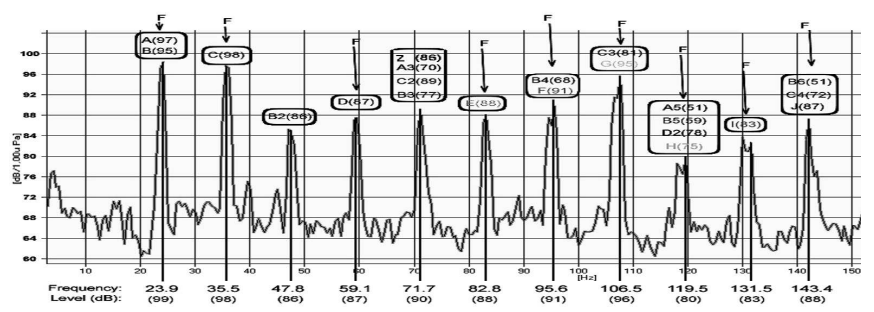

Fig. 6. Analysis of the DFT of K1 at $6 \mathrm{kn}$ in diesel configuration.

To be able to build Fig. 6, it is appropriate to study the frequencies and the amplitudes of the peaks. Without a doubt, it is known that harmonics are multiples of a fundamental hence the frequency study. Moreover, a loss of $9 \mathrm{~dB}$ between harmonics of the same frequency should be observed. First, the study consists in asserting that the first peak as you might expect is a fundamental. As no other frequencies below $23.9 \mathrm{~Hz}$ appear it is a fundamental. Its second harmonic is the $47.8 \mathrm{~Hz}$ frequency, that amplitude must be $9 \mathrm{~dB}$ lower. However, a $13 \mathrm{~dB}$ loss is observed which means a second fundamental and consequently a second source contributes at $23.9 \mathrm{~Hz}$. The level of this new source called B is $95 \mathrm{~dB}$ so that the summation of the levels of the two sources corresponds to the value reached by the peak $99 \mathrm{~dB}$. The same goes for the following peaks; if they are not multiples of the previous fundamentals, they are considered as new sources or if their amplitudes do not succeed in reaching the value that must be found in summing the different contribution of the harmonics which must step in the peak level.

The next figure displays the DFT calculated with $\mathrm{Matlab}^{\circledR}$ for K2 in diesel configuration at $4.5 \mathrm{kn}$. The signals have been computed between -10 and $+10 \mathrm{~s}$, taking for zero the time when the ship is above the probes, and the average DFT has been plotted.

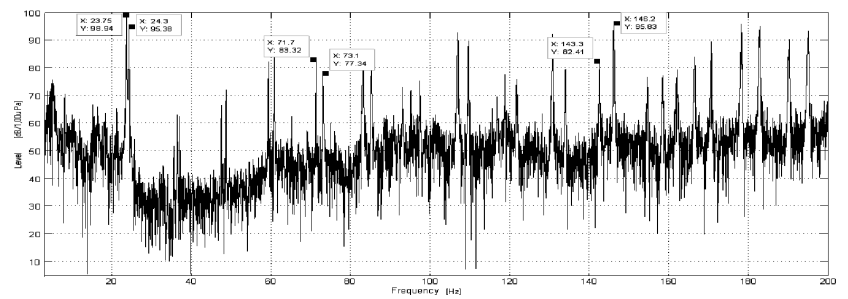

Fig. 7. DFT of the K2 submarine in diesel configuration at $4.5 \mathrm{kn}$.

The following figure displays the spectrogram of K1 at $6 \mathrm{kn}$ for the bandwidth 0 to $200 \mathrm{~Hz}$.

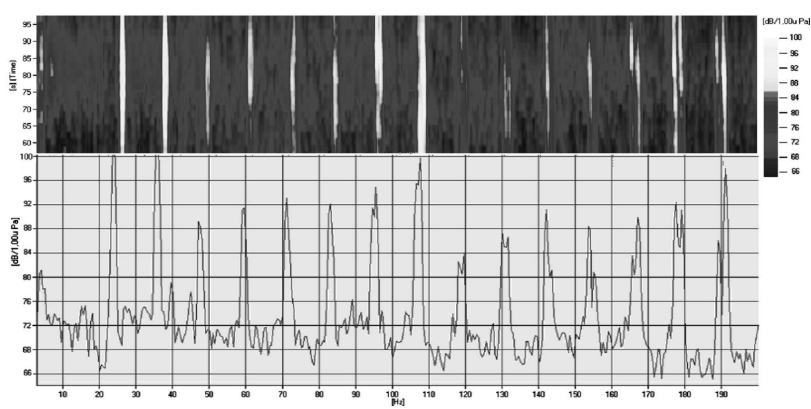

Fig. 8. Spectrograms of the $\mathrm{K} 1$ at $6 \mathrm{kn}$ for the diesel configuration.

In latest years increasingly sophisticated numerical and experimental research on propeller cavitation has been conducted. The main advantage of the short time Fourier transform concerns the cavitation. The DFT gives no clear information about the cavitation contrary to the STFT. It is caused because DFT shows information which, it can be said, is averaged for whole signal, so cavitations effect is not easily observed. Because STFT shows information in time-frequency domain, it presents clearly cavitations in the moment of time when propeller of ship is above the hydrophones. Cavitation in propellers 
occurs in the tip vortex or the gap between the end wall and the blade tip and on the blade surface. The blade surface cavitation takes two main forms: bubbly cavities occurring individually or in clusters on or near the blade surface on its suction side and sheet cavities that extend along the chord from the line of smallest amount of pressure at the leading edge.

The next figure shows, in green, that the cavitation clearly stands out on the spectrogram.

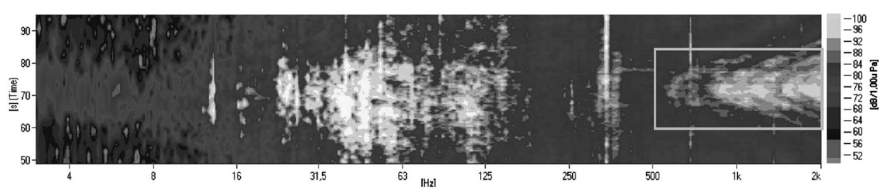

Fig. 9. Logarithmic spectrograms of $\mathrm{K} 1$ at $7.8 \mathrm{kn}$.

Thanks to the different spectrograms, the critical speed of $\mathrm{K} 1$ has been estimated at $7 \mathrm{kn}$ and $\mathrm{K} 2$ cavitates from $6.5 \mathrm{kn}$.

\section{Summary}

The results obtained during measurements have been analyzed and a criticism of each method has been done, focusing on its advantages in the field of the identification of the noise sources. Each method described in this paper has specific advantages. Unfortunately some of these techniques are rapidly limited and cannot make a precise analysis possible. However they are complementary: the disadvantages of one being counterbalanced by the advantages of the others and the conclusion made thanks to one can be confirmed, or not, by the others. Thus the totality of these methods makes an identification of the sources possible because it offers a global vision of the radiated noises.

\section{References}

[1] R.J. Urick, Principles of Underwater Sound, McGraw-Hill, New York 1975.

[2] D. Ross, Mechanics of Underwater Noise, Pergamon, New York 1976.

[3] Royal Norwegian Navy, Brüel \& Kjær application notes.

[4] S.J. Malinowski, I. Gloza, Acta Acoustica united with Acoustica 88, 718 (2002).

[5] M. Crocker, F. Jacobsen, in: Crocker, M. Encyclopaedia of Acoustics, Vol. 4, Ed. M.J. Crocker, John Wiley, New York 1997, p. 1855.

[6] I. Gloza, Acta Phys. Pol. A 118, 58 (2010). 